\title{
Morphometric Studies of the Endodermis in Petioles of Seedlings Grown During a Spaceflight Experiment.
}

\author{
Christina Marie Johnson ${ }^{1}$, Katherine D.L. Millar ${ }^{1,2}$, Richard E. Edelmann ${ }^{1}$, John Z. Kiss ${ }^{1,2}$ \\ ${ }^{1}$ Miami University, Department of Botany, Oxford, OH, 45056 USA \\ 2. University of Mississippi, Department of Biology, Oxford, MS, 38677 USA.
}

Gravity is a constant unidirectional stimulus on Earth, and gravitropism in plants involves specialized subcellular mechanisms. Within the shoots of plants, gravity perception takes place in a tissue layer called the endodermis [1]. Located between the cortex and vasculature, the endodermis contains an abundance of specialized starch-filled organelles called amyloplasts. On Earth, these organelles act as gravity-perceiving statoliths that sediment in-line with the gravity vector.

To investigate the mechanisms of this subcellular machinery in microgravity, we conducted a spaceflight experiment [2]. In April 2010 on the Space Shuttle mission STS-131, seedlings of Arabidopsis thaliana (Landsberg ecotype) were grown in darkness using the Biological Research in Canisters (BRIC) hardware. After 14 days of growth, plants were fixed on-orbit using glutaraldehyde. Seedlings were removed from the hardware after landing on day 15 (Figure 1). An Earth-based ground control took place in the hardware in a growth chamber mimicking the temperature and humidity conditions of the Space Shuttle.

Developing procedures for accurately visualizing the amyloplasts and subcellular structures proved challenging at every step. Post-flight seedlings were processed and sectioned at $1.5 \mu \mathrm{m}$ then post-section stained with Toluidine blue (to distinguish cell walls and nuclei) and Lugol's iodine which stained the starch-filled amyloplasts (Figure 2a). Transmission electron microscopy was used to confirm the presence of starch in petioles (Figure 2b). When sections were viewed using light microscopy, we found an extensive layer of statoliths present in the petiole of the first leaves, or cotyledons, but absent in the endodermis of the hypocotyls of the 14-day-old seedlings.

Image analyses of light micrographs were conducted using Image Pro Plus software version 6.3 to determine differences in the positioning of statoliths in the endodermis of spaceflight and ground control samples. Developing a consistent methodology for image analysis once again proved problematic. Cells with both regular and irregular shapes were chosen for analysis. Images were calibrated to the appropriate scale then brightness, contrast, gain, and color were adjusted to facilitate computer-aided measurements (Figure 3). Automated measurements included cell area, location of cell centroid, minimum/maximum diameter, and all diameters. Additional measurements of the distance from the centroid to each amyloplast were also conducted.

In conclusion we found that dual staining with Lugol's iodine and Toluidine Blue allows distinction between nuclei and amyloplasts. With this staining technique, we saw that while14 day-old plants contain few amyloplasts in the endodermis of hypocotyls, many are contained within the endodermis of petioles. We have developed the methods to begin analysis of the effects of microgravity on plastid location in the gravity-perceiving endodermal cells including automated image analysis of both regular and irregular endodermal cells. While gravitropism of leaves have been investigated through ground 
studies [3] to our knowledge, this is the first study to consider the role of statoliths in the petioles of seedlings grown in microgravity.

References:

[1] M. Toyota, S. Gilroy. American Journal of Botany. 100 (2013), p. 111-125.

[2] K.D.L. Millar, C.M. Johnson, R.E.Edelmann, et al. Astrobiology. 11 2011), p. 787-797.

[3] E. Mano, G. Horiguchi, H. Tsukaya, Plant Cell Physiology. 47 (2006), p.217-223.

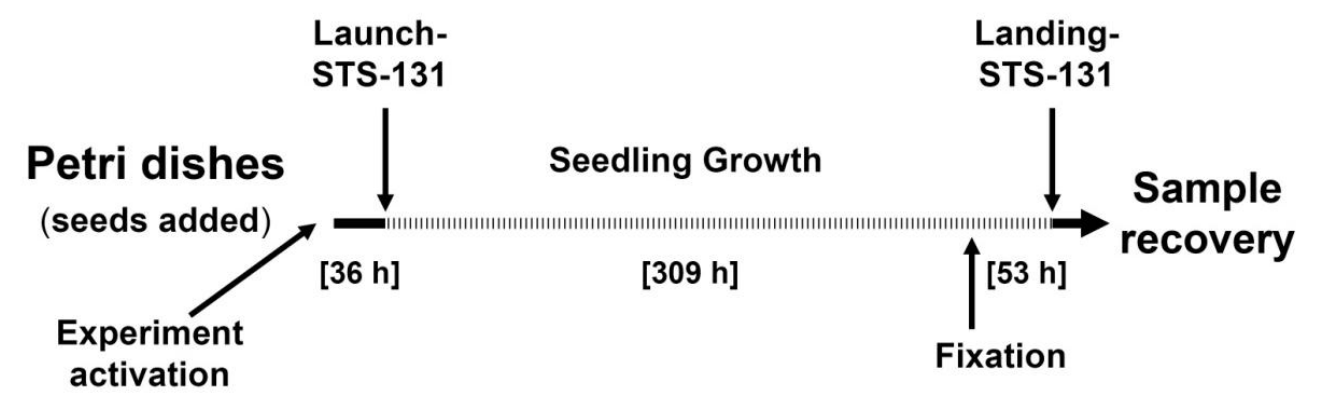

Figure 1: Mission timeline. 36 hours prior to launch, the petri dishes were loaded into the Shuttle. After 309 hours in orbit, fixatives flooded the plates with seedlings that developed during the flight. 53 hours later, the Shuttle landed and samples were recovered.

a.

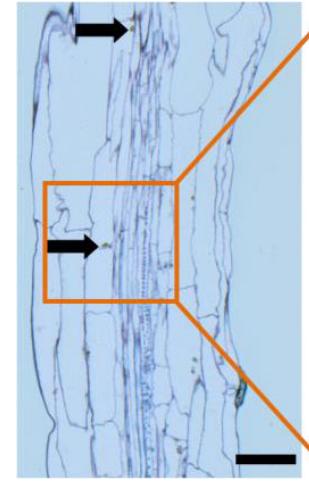

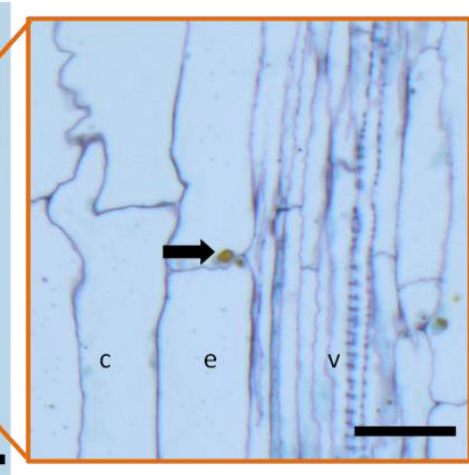

b.

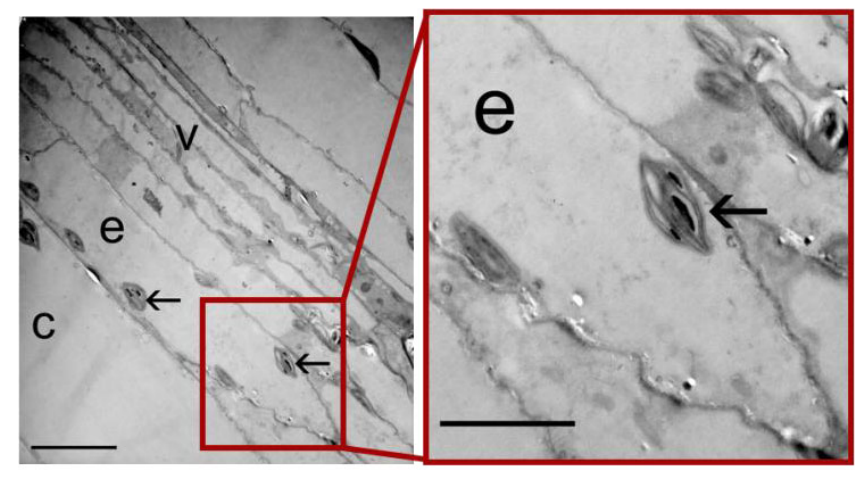

Figure 2: Microscopic images of the seedlings a. Light micrograph of sections stained with Lugol's iodine (IKI) and Toluidine Blue. Samples stained using Toluidine Blue showed excellent distinction of cell walls and nuclei, while IKI boldly showed starch. A combination of these two stains provided the clarity necessary to distinguish between amyloplasts and nuclei. Endodermis (e) is located between vascular (v) and cortical (c) tissues. Arrows indicate amyloplasts. Bars represent $50 \mu \mathrm{m}$ and $25 \mu \mathrm{m}$. b. Transmission Electron Micrograph of petiole. Starch-filled amylopasts (arrow) within endodermis (e), which is a layer of tissue between the vasculature (v) and cortex (c). Bars represent $10 \mu \mathrm{m}$ and $5 \mu \mathrm{m}$.
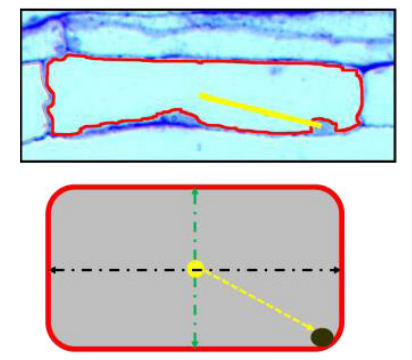
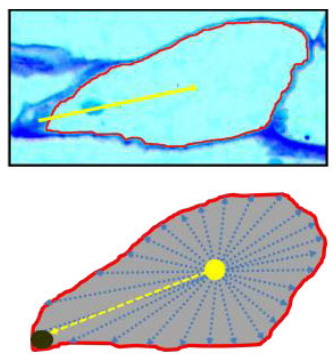

Figure 3: Image analysis of regular and irregular cell shapes. Images were contrast-enhanced to facilitate analysis. Automated measurements included cell area, location of cell centroid (yellow circle), minimum (green line) / maximum (black line) diameter, and all diameters (blue lines). Manual measurements of the distance from the centroid to each amyloplast (yellow line to brown oval) were also conducted. 